\title{
Frequency-Domain Vibration Analysis for Characterizing the Dynamic Mechanical Properties of Materials
}

\author{
P. Raju Mantena \\ Department of Mechanical Engineering \\ The University of Mississippi \\ University, MS 38677
}

\begin{abstract}
Dynamic stiffness and internal damping are referred to as the dynamic mechanical properties of a material and are often expressed in terms of a complex stiffness or complex modulus. The measurement of dynamic mechanical properties of structural materials is of interest for at least two reasons; (a) accurate numerical values of such intrinsic material properties are needed as input to design equations, and (b) dynamic mechanical property measurements can be used for quality control operations during fabrication and/or for periodic in-situ inspection during the service life of the components. The increasing use of composite materials in the aerospace, aircraft and automobile industries has brought about the need for rapid and reliable experimental techniques to characterize their mechanical properties. In the field of vibrations frequency-domain testing is increasingly becoming state-of-the-art as opposed to time-domain experimentation.

In this paper the details of a non-destructive experimental technique for characterizing materials using the impulsive excitation frequency-domain vibration analysis, is described. The relationship between the resonant frequency of vibration and half-power bandwidth on the dynamic mechanical properties of different materials is underscored. Experiments are performed on metallic, polymeric and composite materials to determine their dynamic modulus and loss factor (a measure of damping), and comparison is made with data obtained from the conventional time-domain based free-vibration decay tests. This experiment has been successfully incorporated as a laboratory exercise for the past few years in the undergraduate mechanical engineering curriculum at The University of Mississippi.
\end{abstract}

\section{INTRODUCTION}

Structural members when put into service are susceptible to extraneous vibration. Apart from the static load carried by the design member, it is subjected to fatigue and other mechanical loadings due to vibrations. If the amplitude of vibration is high then the component is likely to experience high induced stresses. Hence, the usefulness of intrinsic material damping to curtail the induced vibration amplitudes is an important design parameter for modeling real world problems. ${ }^{1,2}$ Damping is a measure of the total energy dissipated in any vibrating structure. Damping can be generated within the material of the structure (material damping), by the fluid surrounding the structure (fluid damping) or by the impact and scraping at joints (friction damping). There are a large number of mechanisms which may cause damping; some of these are now well understood, others less so. In solids, material damping attributed to internal friction may be 
produced by several different mechanisms with the mechanical energy being converted into heat. One such process depends on the inelastic behavior of the material, with the thermal losses, however, being more important in the case of metals. Zener ${ }^{3}$ discusses several different thermal mechanisms which result in the dissipation of mechanical energy into heat. In fiber reinforced polymeric matrix materials, it appears that the viscoelastic behavior of the bulk matrix and the friction at the interface caused by relative motion between matrix and fiber are the primary sources of energy dissipation resulting in damping.

The present lack of knowledge concerning damping and the effects of temperature and frequency on it is an example of the gap that often develops between scientific and engineering work. The scientists, in order to simplify the problem for easier understanding, have in general restricted themselves to low stress levels which are primarily of interest in acoustical work. The engineers in taking data they needed for structural work, have largely overlooked some of the important ideas and variables - in this case the temperature and frequency dependence. Here is an area where more work needs to be done and it seems likely that it will not only be of practical value but can if properly planned help to shed light on such problems as the detection of flaws, inhomogeneities and the optimization of material properties. ${ }^{4}$

From the results of material damping measurements, interesting conclusions have been hypothesized by experimental physicists about such things as molecular resonances, dislocation energies, thermal diffusivities and magnetic domain motions. The detection of flaws offers a special field of application for damping measurements. Any inhomogeneity may be expected to increase the damping capacity, since energy should be dissipated at the site of the flaw either as a result of stress concentration or solid friction at crack interfaces during vibration. The size of the defect that can be detected depends on whether the energy dissipated at the flaw is a significant proportion of the total normal energy dissipation in the specimen. A homogeneous isotropic specimen free of stresses and discontinuities has the same value of damping in all directions (e.g. metallic materials). A specimen with stresses or discontinuities such as a fiber reinforced composite has an anisotropy of damping. Damping capacity measurements have been effectively used as a parameter for characterizing materials non-destructively. It has been used successfully to detect the integrity of a bonded joint, surface and internal flaws in materials and for tracking degradation in composites and other advanced materials. ${ }^{4,5}$ Another important mechanical property investigated in this exercise is the stiffness or dynamic modulus of the material which dictates its ability to support loads.

The above discussion has been presented to familiarize the student about the dynamic stiffness and the damping capacity of a material (along with its usefulness to curtail vibration amplitudes), which are generally temperature and frequency dependent. A number of experimental techniques are employed for quantifying the dynamic mechanical properties of materials. All measurement techniques necessarily influence the quantity being measured. When measuring small levels of damping, it is especially important to minimize the influence of the measurement procedure. An experimental technique to determine the dynamic material properties using time-domain vibration analysis is described in Reference 6 in which free vibration decay tests are performed on cantilever beam specimens for determining both the dynamic modulus and loss factor.

The more sophisticated frequency-domain transfer function analysis under impulsive excitation has been utilized for studies on the improvement and optimization of internal damping of fiber reinforced composite materials.7 The purpose of this article is to introduce students to a simple technique for determining the dynamic mechanical properties of materials from impulse frequency response vibration tests. Experiments are performed on four different categories of materials such as epoxy, polyester, aluminum and 
composite specimens to determine the dynamic modulus and loss factor. This provides the student with a good understanding of the dynamic mechanical properties of materials and acquaints the student with the testing procedure. The experiment is simple to understand and the equipment easily portable, facilitating lecture/laboratory usage or for high school demonstrations. This module would be appropriate at the senior undergraduate level as a materials/structures laboratory exercise, with engineering materials and mechanics courses as pre-requisites.

\section{EQUIPMENT}

Equipment needed: HP 35660A spectrum analyzer, HP 9000 personal computer, Kaman KD-2400 displacement measuring system, PCB impulse hammer, precision clamp, HP-IB interface cables.

\section{THEORY}

The vibration response of a material for a given boundary condition is dependent on its mechanical properties. Especially, the stiffness and damping are the two major constituents that influence the characteristics of the vibration response. Vibration analysis which is an established field to test materials non-destructively utilizes this feature quite effectively. In this study, material specimens are excited in the flexural mode to determine the dynamic flexural properties. Other test configurations are possible for obtaining the extensional and torsional dynamic properties, but are beyond the scope of the level at which this exercise is offered. They would be more appropriate for a graduate level laboratory.

The Bernoulli-Euler equation of motion of a body vibrating in the flexural mode with constant flexural stiffness (EI) is used to derive the expression for the frequency of vibration. For a cantilever beam vibrating in the fundamental mode this expression is given by:

$$
f=\frac{(1.875)^{2}}{2 \pi L^{2}} \sqrt{\frac{E_{f} I}{\rho A}}
$$

where $\mathrm{L}=$ free-length of the cantilever beam

$\mathrm{E}_{\mathrm{f}}=$ flexural modulus of elasticity of the beam

$\mathrm{I}=$ area moment of inertia of the beam

$\mathrm{p}=$ mass density of the beam

$\mathrm{A}=$ cross-sectional area of the beam

$\mathrm{f}=$ resonant frequency

\section{TEST PROCEDURE}

Dynamic tests are performed on different material specimens of appropriate dimensions (length $\simeq 20$ $\mathrm{cm}$, width $\simeq 2 \mathrm{~cm}$ and thickness $\simeq 0.3 \mathrm{~cm}$ ) mounted in a cantilever configuration, to determine the dynamic flexural modulus and loss factor. Care should be taken to maintain the appropriate length to thickness ratio 
(at least 16: 1) for exciting the specimen in the flexural mode so as to avoid shear effects, which are noticeable at lower length to thickness ratios. A schematic diagram of the experimental setup for performing the impulse frequency response tests is shown in Figure 1.

A small strip of aluminum foil is attached at the free end of the specimen to facilitate the measurement of displacement using the eddy current proximity probe with the other end firmly clamped between the jaws of the vise. The displacement probe is calibrated to limit the maximum and minimum displacements to within the linear range of the transducer for accurate measurement of data. After making the connections as shown in Figure 1, the transfer function or frequency response function for each material specimen is found by lightly tapping it with the instrumented impact hammer which has a force transducer attached to its head. The specimen response is measured by the non-contacting proximity transducer. The signals from the force transducer and motion transducer are fed to Channels A and B, respectively, of the Fast Fourier Transform based spectrum analyzer which displays the desired transfer function. A typical transfer function showing several resonant peaks is shown in Figure 2. Use of the 'zoom' feature on the spectrum analyzer makes it possible to analyze the individual peaks more accurately. The dynamic flexural modulus, $\mathrm{E}_{\mathrm{f}}$, for each specimen can be computed by rearranging the terms and substituting the fundamental resonant frequency displayed on the screen of the spectrum analyzer into Equation 1.

The material damping for each specimen is obtained by curve fitting the resonant frequency using the half-power bandwidth technique (Figure 3). The half-power points are found on either side of the resonant frequency, $\mathrm{f}$, at $3 \mathrm{~dB}$ below the peak value of the transfer function when a logarithmic magnitude scale is used or at 0.707 of this peak value when a linear magnitude scale is used. The corresponding frequencies $\left(f_{1}\right.$ and $\mathrm{f}_{2}$ ) at these half-power points are substituted into the following expression for the loss factor:

$$
\eta=\frac{\Delta f}{f}=\frac{f_{2}-f_{1}}{f}
$$

All the computations can be done by using appropriate cursors provided on the spectrum analyzer and by performing hand calculations. For better accuracy, however, the displayed spectrums can be digitized, transferred to the computer and analyzed using appropriate curve-fit numerical routines to estimate the dynamic modulus and damping. It is recommended that at least two to three tests are performed on each specimen, with a minimum of three samples tested for each type of material.

\section{DISCUSSION}

Table 1 summarizes the computed dynamic mechanical properties. It should be noted that for each type of material (and boundary conditions) the frequency of vibration is inversely proportional to its free length. Since some of the materials under consideration are viscoelastic in nature (e.g. epoxy), the material properties are dependent on the frequency of vibration. For comparative purposes, the lengths of the test specimens were therefore adjusted (by clamping at different lengths) until the frequency of vibration was approximately the same for all the material specimens.

From Table 1, it is clear that materials having high dynamic flexural modulus or stiffness exhibit low damping capacity and vice versa. This trend is usually observed in most of the conventional materials. The composite materials considered in this exercise are made up of a polymeric matrix material which contributes 
to damping along with the high-modulus reinforcing fibers providing good flexural stiffness. The amount of damping and stiffness can be controlled by the volume fraction and type of fibers and the matrix used for fabricating the composite. The specimens considered in this demonstration were selected to cover a wide range of dynamic modulus and damping values, which is helpful for demonstrating to the students the choice and selection of materials depending on the end application. The criteria for selecting grey cast iron due to its high damping capacity for reducing machinery noise/vibration can be explained using this technique. It can also be used as a non-destructive tool to assess the quality (strength) of an adhesively bonded joint by, monitoring the changes in damping and resonant frequency of the component (increase in debond area results in a sharp increase in damping). ${ }^{5}$ The exercise demonstrates the advantages of using composite structures having good damping and modulus in aerospace applications and other harsh vibratory environments. This frequency domain vibration technique has also been effectively used to characterize and optimize the production of pultruded composite materials. ${ }^{8}$

Table 1. Dynamic mechanical properties determined for various materials

\begin{tabular}{||l|c|c|c||}
\hline \hline \multirow{2}{*}{ Material } & \multirow{2}{*}{$\begin{array}{c}\text { Natural frequency } \\
\text { of vibration }(\mathrm{Hz})\end{array}$} & \multicolumn{2}{c||}{ Dynamic flexural properties } \\
\cline { 3 - 4 } & & Modulus $(\mathrm{GPa})$ & Loss factor \\
\hline Polyester & 51.1 & 3.8 & 0.0339 \\
Epoxy & 50.1 & 4.5 & 0.0135 \\
Aluminum & 51.0 & 67.7 & 0.0015 \\
Graphite/Epoxy & 51.7 & 112.3 & 0.0033 \\
\hline
\end{tabular}

\section{CLOSURE}

A simple experimental technique to characterize materials rapidly and efficiently using frequency domain vibration analysis has been discussed. The relationship between resonant frequency and the halfpower bandwidth on dynamic mechanical properties of different materials has been demonstrated. Selecting materials for a desired end application and the potential use of damping and resonant frequency as nondestructive quality control parameters is underscored. This experiment has been incorporated as a laboratory exercise for the past several years in the undergraduate mechanical engineering curriculum at The University of Mississippi. The results were found to be comparable with data obtained from the time domain based free vibration decay technique. ${ }^{6}$

\section{ACKNOWLEDGMENTS}

Support for this work from the NSF/ILI Division of Undergraduate Education (Grant \# DUE 9351309 ) and the University of Mississippi is greatly appreciated. 


\section{REFERENCES}

1. McTavish, D. J., Hughes, P. C., Soucy, Y. and Graham W. B., "Prediction and Measurement of Modal Damping for Viscoelastic Space Structures, ” J. American Institute of Aeronautics and Astronautics, Vol. 30, No. 5, May 1992, pp 1392-1399.

2. O’Neil, M. and Hollaway, L., "Vibrational Characteristics of Assemblies of Graphite-Reinforced Polyethersulphone Tubes”, Composites, Vol. 21, No. 1, Jan. 1990, pp 13-21.

3. Lazan, B. J., Damping of Materials and Members in Structurual Mechanics, Pergamon Press, 1968

4. Mantena, P. Raju, "NDE Applications of Damping Measurements", Proceedings of NOISE-CON 91, July 14-16, 1991, Tarrytown, NY.

5. Srivatsan, T. S., Mantena, R., Gibson, R. F., Place, T. A. and Sudarshan, T. S., "Electromagnetic Measurement of Damping Capacity to Detect Damage in Adhesively Bonded Material", J. Materials Evaluation, Vol. 47, No. 5, May 1989, pp 564-570.

6. Kowsika V.S.L.N. Murthy and Mantena P. R., "Experimental Technique to Determine Dynamic Material Properties Using Time Domain Vibration Analysis, ” J. Mater. Educ., Vol. 16, pp. 73-84, 1994

7. Mantena, P. Raju and Gibson, R. F., "Dynamic Mechanical Properties of Hybrid Polyethylene/Graphite Composites, " Proceedings of the 22nd International SAMPE Technical Conference, November 6-8, 1990, pp. 370-382.

8. P. Raju Mantena, Vangipuram R., and Vaughan J. G., "Dynamic Flexural Properties of Pultruded Glass/Graphite Hybrid Composites, " Proceedings of the 39th International SAMPE Symposium and Exhibition, April 11-14, 1994, vol. 39, pp. 174-182.

\section{BIOGRAPHICAL SKETCH OF THE AUTHOR}

P. Raju Mantena received a BE (1973) in Mechanical Engineering from Andhra University and a post-graduate diploma in Design Engineering (1975) from the Indian Institute of Technology, Delhi. He worked for eight years as a Development Engineer in BHEL, Corporate Research and Development Laboratories, Hyderabad, India. Dr. Mantena received his MS (1985) and PhD (1989) in Mechanical Engineering from the University of Idaho, after which he was employed as a research associate in Wayne State University, Detroit. Presently he is an assistant professor in the Department of Mechanical Engineering at the University of Mississippi teaching design, vibrations, mechanics of composite materials, and experimental stress analysis. His research interests are in optimizing the dynamic properties of composites and nondestructive evaluation. He has published extensively in these areas, organized and served as session chair in international conferences, and is an active member of ASM, ASME and SAMPE. 


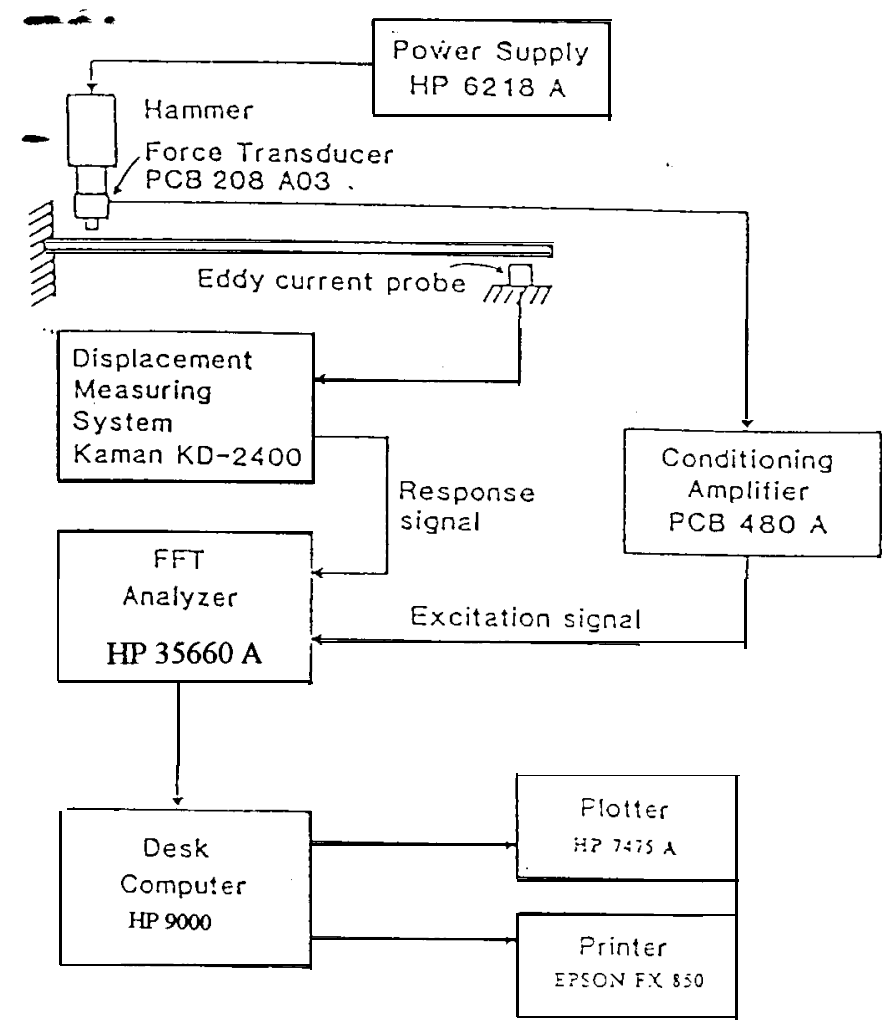

Figure 1, Impulse frequency response test apparatus for flexural vibration

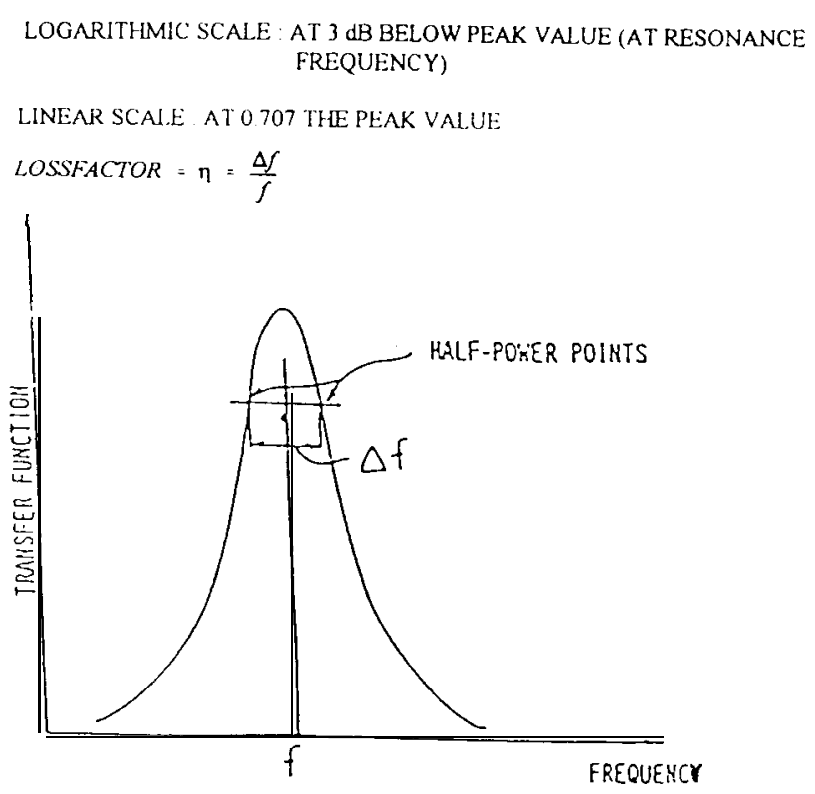

Figure 3. Half-power bandwidth method for computing loss factor

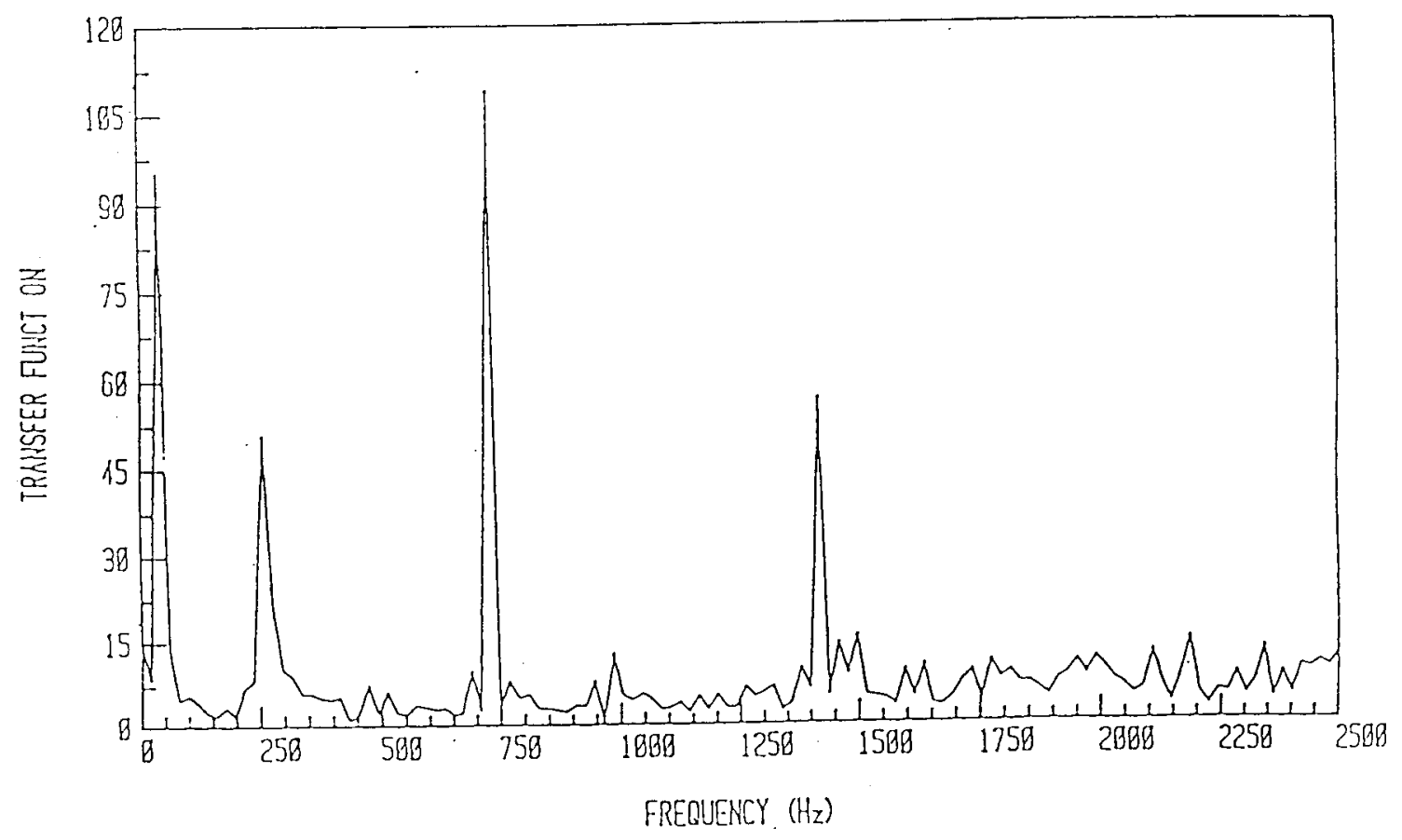

Figure 2. Typical specimen transfer function vs frequency (or the frequency response curve) 